\title{
Short communication: Reliability of single-step genomic BLUP breeding values by multi-trait test-day model analysis
}

\author{
J. Bauer, ${ }^{1}$ J. Přibyl, and L. Vostrý \\ Institute of Animal Science, Přátelství 815, 10401 Praha-Uhříněves, Czech Republic
}

\begin{abstract}
The purpose of our study was to develop an approximation procedure to estimate reliabilities of single-step genomic BLUP breeding values in a test-day model for routine evaluation of milk yield in a dairy cattle population. Input data consisted of 20,220,047 first-, second-, and third-lactation test-day milk yield records of 1,126,102 Czech Holstein cows (each lactation being considered a separate trait), with $1,844,679$ animals in the pedigree file and with genomic data from 2,236 bulls. Evaluation was according to a multi-lactation model. The procedure was based on the effective number of records per animal from milk recording as well as from genomic and pedigree relationships. Traits were analyzed individually, and genetic covariances among traits were subsequently taken into account. The use of genomic information increased average reliability in young bulls from 0.276 to 0.505 , but increased reliability in proven bulls only from 0.828 to 0.855 . The reliabilities of genomic breeding values in multi-trait evaluation for first, second and third lactations, respectively, averaged $0.652,0.673$, and 0.633 for young bulls and $0.907,0.894$, and 0.852 for proven bulls. For an index combining all 3 lactations, the average reliability of a single-step genomic BLUP prediction was 0.712 and 0.925 for younger and proven bulls, respectively. Increased reliability due to genotyping in the population of all genotyped and nongenotyped animals was very small $(<0.01)$ because of the small proportion of genotyped animals in the population.
\end{abstract}

Key words: reliability, genomic prediction, singlestep genomic BLUP, test-day model

\section{INTRODUCTION}

Utilizing information from genomic breeding values is a promising procedure to increase accuracy of genetic evaluations of farm animals, especially from young animals without performance data. Two types of genomic

Received January 22, 2015.

Accepted March 20, 2015.

${ }^{1}$ Corresponding author: bauer.jiri@vuzv.cz prediction of breeding values are currently used: the multi-step method of Meuwissen et al. (2001) and VanRaden (2008) and the single-step method of Misztal et al. (2009) and Christensen and Lund (2010).

Reliabilities of genomic breeding values are required to achieve efficient selection of parents for future generations, especially for the international Interbull multiple across-country evaluation (MACE), in which reliabilities are used as weighting factors for various information sources (Schaeffer, 1994; Sullivan and Jakobsen, 2012). They can be calculated by inversion of the left hand side of a BLUP system of equations (VanRaden, 2008) but this is usually not feasible due to large population sizes and massive computational requirements. For this reason, methods to approximate reliabilities of genomic EBV (GEBV) were developed by Szyda et al. (2011) for the multi-step method and by Misztal et al. (2013) for the single-step procedure.

Přibyl et al. $(2012,2013)$ and Zavadilová et al. (2014) developed a workable procedure for genomic evaluation of dairy cattle in the Czech Republic. However, for routine use, it is necessary to develop a method to calculate reliabilities of genomic breeding values. The aim of our current investigation, therefore, was to develop a method for routine approximation of the reliability of single-step genomic breeding values for test-day model evaluation.

We used 20,220,047 test-day milk yield (kg) records of 1,126,102 Czech Holstein cows. Observations were from the first 3 lactations, with each considered as a separate trait $(9,480,924,6,681,967$, and 4,057,156 first-, second-, and third-lactation records, respectively). With inclusion of 6 generations of ancestors, the total number of animals in the evaluation was $1,844,679$. There were 2,236 genotyped bulls in the population, of which 445 were considered unproven "young" bulls (fewer than 3 offspring each) and the rest were considered proven. The proven bulls had 240 daughters on average.

The Illumina BovineSNP50 Beadchip V2 (Illumina Inc., San Diego, CA) was used for genotyping. To eliminate possible input errors, data were edited for minor allele frequency $(\mathrm{MAF})<0.05$, number of loci per bull $<90 \%$ of all possible loci, number of bulls per locus $<90 \%$ of all possible bulls and large discrepancy 
of pedigree relationship $\mathbf{A}_{22}$ with genomic relationship G (absolute difference in relationship to others $>3$ animals $>0.30$ ), and proportion of Holstein genes $<85 \%$. After filtering, 40,653 SNP loci were used in the analysis.

The routine random regression test-day model of animal evaluation in the Czech Republic is described in studies of Zavadilová et al. (2005a,b) and evaluates cow test-day milk production by fixed effects: herd-test-day, Legendre polynomial regression coefficients for lactation and contemporary group, regressor for lactation day, and random effects of Legendre polynomials for permanent environment and additive genetic effect of animal.

We developed our own programs for prediction of genomic breeding values and approximation of reliabilities parallel in 2 languages: SAS (SAS Institute, 2005) and COBOL (ISO, 2014).

In routine BLUP evaluation (EBV prediction), a pedigree relationship matrix $\mathbf{A}$ is used to quantify the additive genetic effect, whereas, for single-step genomic (ssGBLUP) evaluation (GEBV prediction), a relationship matrix $\mathbf{H}$ is used, in which is $\mathbf{A}$ augmented by a genomic relationship matrix $\mathbf{G}$, with weights 20 and $80 \%$ respectively (Christensen and Lund, 2010). Matrix $\mathbf{G}$ is constructed according to deviations from the averages of observed allele frequencies and standardized so the average of diagonals equals 1 (Forni et al., 2011) and then shifted so the elements of the pedigree relationship matrix of genotyped animals $\mathbf{A}_{22}$ and elements of $\mathbf{G}$ will have the same average (Vitezica et al., 2011).

Overall GEBV or EBV were calculated in our current case as an index (i) by summing values of genetic polynomials for $300 \mathrm{~d}$ of lactation and 3 lactations, and then calculating the average across those 3 lactations:

$$
\mathbf{i}=\left(\Sigma \Sigma \mathrm{a}_{z j m} \times \mathrm{v}_{m t}\right) / 3,
$$

where $\Sigma \Sigma=$ sum of random Legendre polynomial regression coefficients $m$ and lactations $j, \mathrm{a}_{z j m}=m$ th random effect of Legendre polynomial regression coefficient for animal $z$ in lactation number $j$ with covariance matrix $(12 \times 12)$ covering random regression coefficients over all 3 lactations and connected to relationship matrix, and $\mathrm{v}_{m t}=m$ th regressor for lactation day $t(t=6, \ldots$, $305)$.

Reliabilities of predicted genomic breeding values were estimated based on the procedure of Misztal et al. (2013). To take into account the multi-trait nature of the evaluation, we used the method described by Strabel et al. (2001). The entire procedure can be characterized as a sequence of the following consecutive steps:
1. Approximation of the reliability of BLUP breeding values for all animals and for each trait by the iterative approach of Misztal et al. (1993). This method is based on the approximation of reliability using the effective number of records arising from performance observations within individual contemporary groups and from relationships among animals in the pedigree. To take into account a permanent environmental effect common for all test-days of the same individual, the effective number of records $\mathbf{d}$ for each trait was updated before the iterative procedure by the formula $\mathbf{d}^{*}=\tau \mathbf{d} /(\tau+\mathbf{d})$ (Misztal et al., 1991), where the asterisk is differentiation mark between $\mathbf{d}^{*}$ and nonupdated $\mathbf{d}$ and $\tau$ is the ratio of residual variance to permanent environmental variance. For this step of the procedure, genetic parameters were averaged throughout individual lactations, and observations were taken into account as repeated records.

2. Calculation of the reliability of genotyped animals with inclusion of the increase due to the additional genomic information in prediction of ssGBLUP genomic breeding values. The calculation was done as follows: reliability $r_{i}^{2}=1-\alpha q_{i i}$, where $\alpha$ is the ratio of error variance to animal genetic variance and $q_{i i}$ is the diagonal elements of the $\mathbf{Q}^{-1}$ matrix:

$$
\mathbf{Q}^{-1}=\left[\mathbf{D}+\left(\mathbf{I}+\mathbf{G}^{-1}-\mathbf{A}_{22}^{-1}\right) \alpha\right]^{-1},
$$

where $\mathbf{D}$ is the contribution of the records and pedigrees to the reliability from step (1), I is the identity matrix, $\mathbf{G}^{-1}$ is the inverse of the genomic relationship matrix, and $\mathbf{A}_{22}^{-1}$ is the inverse of the section of the pedigree-based relationship matrix that contains relationship information from only the genotyped animals (Misztal et al., 2013). We applied this procedure for each trait separately.

3. Addition of the contribution of genotyping to the reliabilities of nongenotyped animals. This step was completed using a procedure similar to that described in the first step. The reliabilities of the genotyped bulls were kept constant to avoid double counting the contribution of the relationships among these bulls and to avoid changing the already fully conveyed values.

4. Re-evaluation of individual reliabilities of breeding values due to the covariances among traits in the multi-trait model by the procedure of Strabel et al. (2001). This method approximates reli- 
Table 1. Average values for genetic parameters ${ }^{1}$ derived from routine Czech dairy cattle evaluation (Zavadilová et al., 2005a)

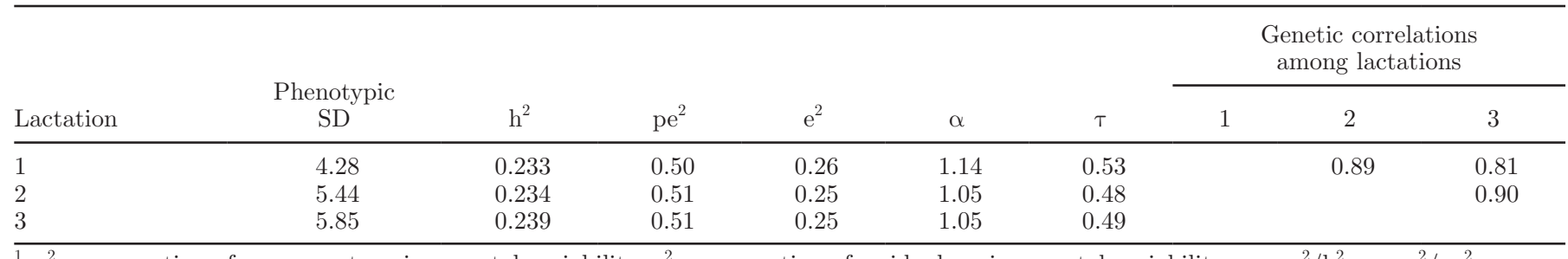

${ }^{1} \mathrm{pe}^{2}=$ proportion of permanent environmental variability; $\mathrm{e}^{2}=$ proportion of residual environmental variability; $\alpha=\mathrm{e}^{2} / \mathrm{h}^{2} ; \tau=\mathrm{e}^{2} / \mathrm{pe}^{2}$.

abilities of breeding values of animals in trait $i$ $\left(r_{i}^{2}\right)$ in the multi-trait model from effective number of records for individual traits as follows:

$$
r_{i}^{2}=1-\left(\hat{\mathbf{C}}^{-1}\right)_{i i} /\left(\mathbf{G}_{0}\right)_{i i}
$$

where $\mathbf{G}_{0}$ is the variance-covariance matrix of the additive genetic effect and $\hat{\mathbf{C}}^{-1}$ is the inversion of the approximation of the left hand side of the mixed-model equations for the animal and its progeny:

$$
\begin{aligned}
\hat{\mathbf{C}} & =\left(\mathbf{O}^{0.5} \mathbf{R}_{0} \mathbf{O}^{0.5}\right)^{-1}+\mathbf{G}_{0}^{-1}+ \\
& \sum_{j}\left[m_{j} \times \frac{\mathbf{G}_{0}^{-1}}{3}-m_{j} \times \frac{2 \mathbf{G}_{0}^{-1}}{3}\left(\frac{4 \mathbf{G}_{0}^{-1}}{3}+\mathbf{R}_{0_{j}}^{-1}\right)^{-1} \frac{2 \mathbf{G}_{0}^{-1}}{3}\right],
\end{aligned}
$$

where $m_{j}$ is number of progeny with missing trait combination $j, \mathbf{O}=\operatorname{diag}\left\{E D C_{i}\right\}$, where $E D C_{i}$ is the effective number of records in trait $i$, and $\mathbf{R}_{0}$ is the variance-covariance matrix for residual effects. Values for $\mathbf{G}_{0}$ and $\mathbf{R}_{0}$ among traits were calculated from corresponding variance covariance matrices of 12 regression coefficients (Zavadilová et al., 2005a). Average values for genetic parameters are summarized in Table 1. Reliability of the 3-lactation index $\left(\operatorname{Rel}_{\mathrm{I}}\right)$ was calculated by selection index procedures, taking into account single-trait reliabilities of individual lactations and covariances among traits:

$$
\operatorname{Rel}_{\mathrm{I}}=\left(\mathbf{b}^{\prime} \mathbf{P b}\right) /\left(\mathbf{a}^{\prime} \mathbf{G}_{\mathrm{T}} \mathbf{a}\right)
$$

where $\mathbf{b}=$ vector of weights in index for 3 lactations; $\mathbf{P}=$ covariance matrix of breeding values; $\mathbf{a}=$ vector of weights of genetic values $(1 / 3$, $1 / 3,1 / 3$ ); and $\mathbf{G}_{\mathbf{T}}=$ genetic covariance matrix among lactations.

Heritabilities for the individual traits calculated from variance and covariance matrices of regression groups were $0.233,0.234$, and 0.239 for the first, second and third lactations, respectively, and $\alpha$ (i.e., ratio of error variance to additive genetic variance) was $1.13,1.04$, and 1.05 respectively. Reliability approximations for nongenomic and genomic contributions to breeding

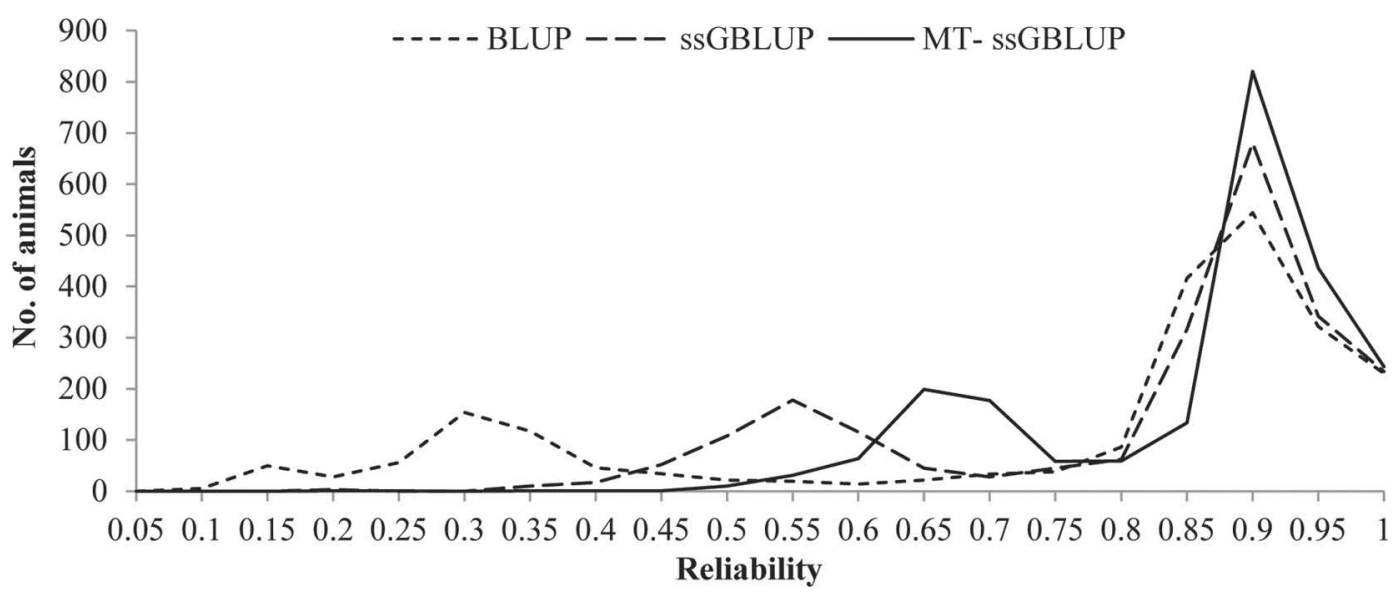

Figure 1. Number of genotyped bulls (regardless of number of daughters) in first-lactation trait by reliability of EBV or genomic EBV in different parts of the procedure: BLUP = single-step genomic BLUP and multi-trait single-step genomic BLUP; ssGBLUP = single-step genomic BLUP; MT-ssGBLUP = multi-trait single-step genomic BLUP. 


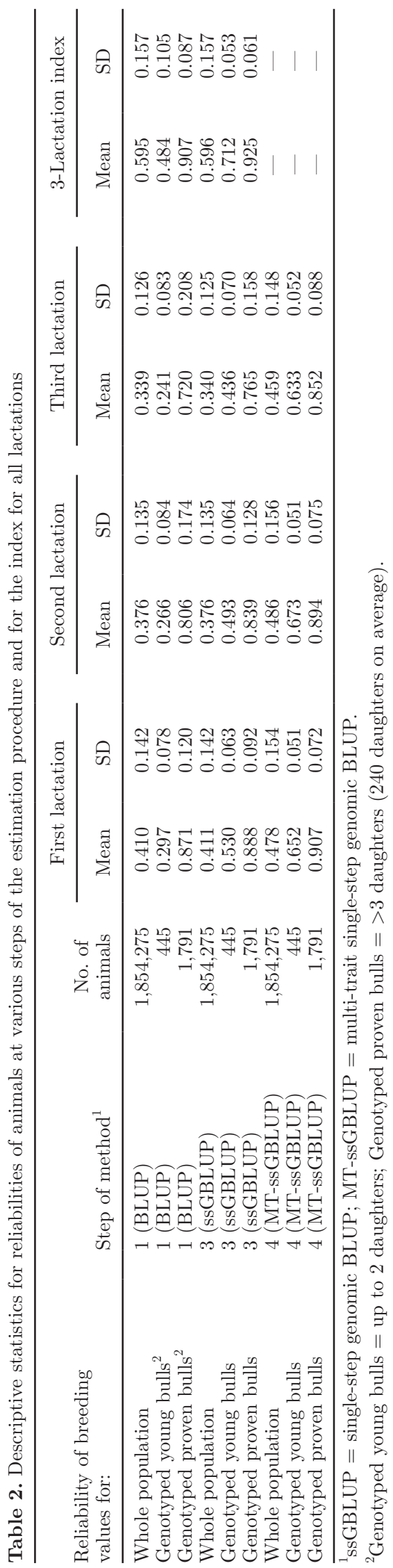

value predictions are shown in Table 2 for the entire population and the 2 categories of genotyped bulls. The distributions of reliabilities of breeding values for BLUP, ssGBLUP, and multi-trait single-step genomic steps of the method were similar among traits. Numbers for the first lactation trait are shown in Figure 1.

The reliability of genomic breeding values in young bulls was distinctly higher than that of breeding values estimated without consideration of genomic information, by an average of 0.229 . This increase is considerably higher than the increase of 0.02 reported by Bauer et al. (2014) using a similar method. The reason for this substantial difference between the 2 reports may be the difference between them in proportion of genotyped proven bulls that can be used as reference population. This is because the reliability of genomic breeding values is approximately linearly dependent upon the number of reference bulls (VanRaden et al., 2009). The number of genotyped proven bulls in the Bauer et al. (2014) study was 217. Moreover, analytical models differed in that the lactation model in the previous study was succeeded in our investigation by a test-day model. In addition, the database of pedigrees from domestic and Interbull files and performance testing records were thoroughly inspected for errors before our analysis, and new information was included correcting the generations of ancestors in Interbull and Czech Moravian Breeders Association (http://www.cmsch.cz) data. The increase in reliability for young bulls in our study was in agreement with Schaeffer (2006), who reported that reliabilities of young animals without performance records could increase due to accessibility of genomic information to approximately 0.65 . An average reliability of 0.48 was reported by Pribyl et al. (2014), after they validated reliabilities in the same population in ssGBLUP by calculation of correlations among predictors of genetic merit for 140 young bulls that had no daughter records in 2008 but had >50 daughter records in 2012 . Our average reliability was higher, but we had more genotyped proven bulls (1,791 vs. 1,259), and Přibyl et al. (2014) used a lactation model with data from only first lactations.

The main advantage of genomic multi-trait evaluation is the inclusion of genetic correlations among traits (Calus and Veerkamp, 2011). Increase in the reliability of GEBV is highest in low-heritability traits that are correlated with high-heritability traits; however, the high-heritability trait benefits from the connection only negligibly (Jia and Jannink, 2012; Hayashi and Iwata, 2013). For this reason, reliabilities in high-heritability traits may be similar to those of low-heritability traits, as reported by $\mathrm{Su}$ et al. (2010) for milk and fertility traits in Danish Holstein population. Application of a multi-trait model in genomic evaluation also increases 
reliabilities of traits with missing phenotype records (Guo et al., 2014).

In our study, heritabilities of traits were too similar to detect differences; however, the greater increase in reliability in traits with fewer records due to the multi-trait evaluation was noticeable (in young bulls, the increase was $0.12,0.18$, and 0.20 in first, second and third lactations, respectively). The increase in reliability of breeding values for the entire population resulting from genotypic information was only 0.01 in our study, largely because of the small proportion of genotyped animals $(2,236$ out of $1,852,039)$.

The method described above was accepted for routine national evaluation of dairy cattle in the Czech Republic in 2014. Genetic evaluation results for milk production traits and their reliabilities are published at http://www.plemdat.cz and are continuously updated.

\section{ACKNOWLEDGMENTS}

This study was supported by the Ministry of Agriculture of the Czech Republic (Prague; project nos. QJ1510139 and MZE RO0714).

\section{REFERENCES}

Bauer, J., L. Vostrý, A. Svitáková, and L. Zavadilová. 2014. Approximation of the reliability of single-step genomic breeding values for dairy cattle in the Czech Republic. Anim. Sci. Pap. Rep. 32:301-306.

Calus, M. P. L., and R. F. Veerkamp. 2011. Accuracy of multi-trait genomic selection using different methods. Genet. Sel. Evol. 43:26.

Christensen, O. F., and M. S. Lund. 2010. Genomic prediction when some animals are not genotyped. Genet. Sel. Evol. 42:2.

Forni, S., I. Aguilar, and I. Misztal. 2011. Different genomic relationship matrices for single step analysis using phenotypic, pedigree and genomic information. Genet. Sel. Evol. 43:1.

Guo, G., F. Zhao, Y. Wang, Y. Zhang, L. Du, and G. Su. 2014. Comparison of single-trait and multiple-trait genomic prediction models. BMC Genet. 15:30.

Hayashi, T., and H. Iwata. 2013. A Bayesian method and its variational approximation for prediction of genomic breeding values in multiple traits. BMC Bioinformatics 14:34.

ISO. 2014. ISO/IEC 1989:2014 Information technology-Programming languages, their environments and system software interfaces-Programming language COBOL. International Organization for Standardization (ISO), Geneva, Switzerland.

Jia, Y., and J.-L. Jannink. 2012. Multiple-trait genomic selection methods increase genetic value prediction accuracy. Genetics 192:1513-1522.

Meuwissen, T. H. E., B. J. Hayes, and M. E. Goddard. 2001. Prediction of total genetic value using genome-wide dense marker maps. Genetics 157:1819-1829.

Misztal, I., T. J. Lawlor, T. H. Short, and G. R. Wiggans. 1991. Continuous genetic evaluation of Holsteins for type. J. Dairy Sci. 74:2001-2009.
Misztal, I., A. Legarra, and I. Aguilar. 2009. Computing procedures for genetic evaluation including phenotypic, full pedigree, and genomic information. J. Dairy Sci. 92:4648-4655.

Misztal, I., A. Legarra, and T. H. Short. 1993. Implementation of single- and multiple-trait animal models for genetic evaluation of Holstein type traits. J. Dairy Sci. 76:1421-1432.

Misztal, I., S. Tsuruta, I. Aguilar, A. Leggara, P. M. Van Raden, and T. J. Lawlor. 2013. Methods to approximate reliabilities in singlestep genomic evaluation. J. Dairy Sci. 96:647-654.

Přibyl, J., J. Bauer, P. Pešek, J. Přibylová, L. Vostrý, and L. Zavadilová. 2014. Domestic and Interbull information in single step genomic evaluation of Holstein milk production. Czech J. Anim. Sci. 59:409-415.

Přibyl, J., J. Haman, T. Kott, J. Přibylová, M. Šimečková, L. Vostrý, L. Zavadilová, V. Čermak, Z. Růžicka, J. Šplíchal, M. Verner, J. Motyčka, and L. Vondrášek. 2012. Single-step prediction of genomic breeding value in a small dairy cattle population with strong import of foreign genes. Czech J. Anim. Sci. 57:151-159.

Přibyl, J., P. Madsen, J. Bauer, J. Přibylová, M. Šimečkova, L. Vostrý, and L. Zavadilová. 2013. Contribution of domestic production records, Interbull estimated breeding values, and single nucleotide polymorphism genetic markers to the single-step genomic evaluation of milk production. J. Dairy Sci. 96:1865-1873.

SAS Institute. 2005. SAS/STAT 9.1 User's Guide. SAS Institute Inc., Cary, NC.

Schaeffer, L. R. 1994. Multiple-country comparison of dairy sire. J. Dairy Sci. 77:2671-2678.

Schaeffer, L. R. 2006. Strategy for applying genome-wide selection in dairy cattle. J. Anim. Breed. Genet. 123:218-223.

Strabel, T., I. Misztal, and J. K. Bertrand. 2001. Approximation of reliabilities for multiple-trait model with maternal effects. J. Anim. Sci. 79:833-839.

Su, G., B. Guldbrandtsen, V. Gregersen, and M. Lund. 2010. Preliminary investigation on reliability of genomic estimated breeding values in the Danish Holstein population. J. Dairy Sci. 93:11751183.

Sullivan, P. G., and J. H. Jakobsen. 2012. Robust GMACE for young bulls-Methodology. Interbull Bull. 45:3-7.

Szyda, J., A. Zarnecki, T. Suchocki, and S. Kaminski. 2011. Fitting and validating the genomic evaluation model to Polish HolsteinFriesian cattle. J. Appl. Genet. 52:363-366.

VanRaden, P. M. 2008. Efficient methods to compute genomic predictions. J. Dairy Sci. 91:4414-4423.

VanRaden, P. M., C. P. Van Tassell, G. R. Wiggans, T. S. Sonstegard, R. D. Schnabel, J. F. Taylor, and F. S. Schenkel. 2009. Invited review: Reliability of genomic predictions for North American Holstein bulls. J. Dairy Sci. 92:16-24.

Vitezica, Z. G., I. Aguilar, I. Misztal, and A. Legarra. 2011. Bias in genomic predictions for populations under selection. Genet. Res. (Camb.) 93:357-366.

Zavadilová, L., J. Jamrozik, and L. R. Schaeffer. 2005a. Genetic parameters for test-day model with random regressions for production traits of Czech Holstein cattle. Czech J. Anim. Sci. 50:142154.

Zavadilová, L., E. Němcova, and J. Wolf. 2005b. Definition of subgroups for fixed regression in the test-day animal model for milk production of Holstein cattle in the Czech Republic. Czech J. Anim. Sci. 50:7-13.

Zavadilová, L., J. Přibyl, L. Vostrý, and J. Bauer. 2014. Single-step genomic evaluation for linear type traits of Holstein cows in Czech Republic. Anim. Sci. Pap. Rep. 32:201-208. 